\title{
On the change points of mean residual life and failure rate functions for some generalized gamma type distributions
}

\author{
M. Parsa*, G.R. Motashami Borzadaran, and A.H. Rezaei Roknabadi \\ Ferdowsi University Of Mashhad (FUM), Azadi Sq., Mashhad, Khorasan Razavi, Iran
}

Received: 18 July 2013 / Accepted: 20 October 2013

\begin{abstract}
Mean residual life and failure rate functions are ubiquitously employed in reliability analysis. The term of useful period of lifetime distributions of bathtub-shaped failure rate functions is referred to the flat rigion of this function and has attracted authors and researchers in reliability, actuary, and survival analysis. In recent years, considering the change points of mean residual life and failure rate functions has been extensively utelized in determining the optimum burn-in time. In this paper we investigate the difference between the change points of failure rate and mean residual life functions of some generalized gamma type distributions due to the capability of these distributions in modeling various bathtub-shaped failure rate functions.
\end{abstract}

Keywords: Change point, failure rate function, mean residual life function, generalized gamma, useful period

\section{Introduction}

Reliability usually refers to the probability that a component or system operates satisfactorily at certain length of time in which it is required. The gamma distribution has a wide application in reliability and survival analysis regarding to its ability of modeling both monotone and non-monotone failure rate functions. Recently, different generalized or modified forms of gamma type distributions have been inspected and used.

Cordeiro et al. [1] introduced exponentiated generalized gamma (EGG) distribution with four parameters, which is flexible to model bathtub shaped failure rate functions. Besides, including sub-models like generalized gamma (GG) distribution [2], exponentiated Weibull (EW) distribution [3,4], exponentiated exponential (EE) distribution $[5,6]$, generalized Rayleigh (GR) distribution [7], etc.

The Kumaraswamy (Kum) distribution [8] is not very popular in statistical works although it can be widely used in modeling lifetime data. Jones [9] has explored its background and compared some properties of Kum and beta distributions. Its cumulative distribution function (cdf) is: $F(x)=1-\left(1-x^{\lambda}\right)^{\phi}$, while $0<x<\infty$ and $\lambda, \phi>0$. Cordeiro and de Castro [10] indicated that if $G(x)$ is cdf of a random variable, the cdf of Kum-generalized (KumG) distribution is defined by:

$$
F(x)=1-\left(1-G(x)^{\lambda}\right)^{\phi} .
$$

\footnotetext{
* Correspondence: grmohtashami@um.ac.ir
}

Very recently, Pascoa et al. [11] introduced Kumaraswamy generalized gamma (KumGG) distribution with five parameters, letting $G(x)$ be the cdf of gamma distribution. This distribution is flexible for modeling bathtub-shaped failure rate functions. The EGG distribution and all of its sub-models could be derived from KumGG distribution as well. Pascoa et al. [11] illusterated the superiority of KumGG to some of its sub-models. Using lifetime of 50 components, they reported smaller akaike information criterion (AIC) and Baysian information criterion (BIC) values for KumGG model among all fitted models. Also serum-reversal time of 148 childeren contaminated with HIV from vertical transmission are studied in [11] and KumGG model has the lowest AIC and BIC statistics.

In a vast range of research area such as reliability, actuarial sciences and survival analysis, the relationship between the failure rate $(\mathrm{FR})$ and mean residual life (MRL) functions and their corresponding change points of bathtub-shaped FR distributions has been investigated by many authors. Gupta and Akman [12] studied the shapes of the functions and their opposite trends. Mi [13] considered the turning points of FR and MRL functions. Lim and Park [14] explored the trend change of continuous MRL. Mitra and Basu [15] developed general estimation of the change points for non-monotonic ageing models based on FR and MRL functions. Salvia [16] analyzed specific situations on discrete MRL. Guess and Proschan [17] discussed the trend changes of FR and MRL functions. Ghai and Mi [18] derived sufficient conditions for the unimodal MRL to imply the bathtub-shaped failure rate. Tang et al. [19] looked into the general characteristics of FR and MRL 
for both continuous and discrete lifetime functions. Chen et al. [20] provided constructing methods of approximate interval estimation for the change point of $\mathrm{FR}$ function. Bekker and Mi [21] pointed out and corrected some errors in the literature by giving counter examples and new proofs and a more complete discussion on the shape of MRL function when the associate FR function is of rollercoaster shape. Mi [22] developed a general approach of the shape of FR and MRL functions. Bebbington et al. [23] estimated the turning point for MRL of a bathtub-shaped failure distribution. Shen et al. [24] examined the change point of FR and MRL functions for series and parallel systems and compared the location of the change points under increasing the number of components in systems. Recently, Shafaei et al. [25] studied the change point of the MRL of some weighted models and showed that the time maximizing the MRL of the greatest order statistic precedes the time maximizing the MRL of baseline model. They also proved that the time maximizing the MRL of proportional failure rate model increases as the probability parameter gets larger values.

Among all papers which studied MRL and FR functions and their trends, a few investigated the flatness of bathtub curve in bathtub-shaped FR distributions which could determine the useful period of the unit or system. Xie et al. [26] considered the difference between the FR function and MRL function change points for some generalized Weibull distributions, since the larger difference between the change points results the longer flat portion and also the longer useful lifetime period. Later, Bebbington et al. [27] proposed a tractable mathematical definition for the "useful period" of lifetime distributions with bathtubshaped FR functions and Bebbington et al. [28] constructed an empirical estimator for MRL change piont and developed a theory for difference between FR and MRL change points.

In this paper, we consider the difference between the change points of FR and MRL functions that gives a reliable idea of the useful lifetime for some generalized gamma type distributions: KumGG disribution, EGG distribution and two of its sub-models which are three-parameter exponentiated gamma (EG3) and two-parameter exponentiated gamma (EG2). Besides, analyze the influence of parameters values on the differences of the change points while they are bathtub-shaped FR distributions.

\section{Preliminaries and some generalized gamma type distributions}

If $F(t)$ and $f(t)$ respectively represent the cumulative density function (cdf) and probability density function (pdf) for $t \geqslant 0$ as a lifetime random variable, the reliability function and FR functions respectively are $\bar{F}(t)=1-F(t)$ and $h(t)=\frac{f(t)}{F(t)}$. The MRL function $m(t)$ states the expected remaining lifetime while the product has survived up to time $t$, and is mathematically expressed as:

$$
m(t)=E[T-t \mid T \geqslant t]=\frac{\int_{t}^{\infty} \bar{F}(x) d x}{\bar{F}(t)} .
$$

According to the definition, $m(0)=\mu$, which is the mean of the distribution. As was discussed in Muth [29], there exists a relationship between MRL and FR functions expressed in the following formula

$$
h(t)=\frac{m^{\prime}(t)+1}{m(t)} .
$$

Also, Calabria and Pulcini [30] showed that

$$
\lim _{t \rightarrow \infty} m(t)=\lim _{t \rightarrow \infty} \frac{1}{h(t)},
$$

while the limit of the latter exists and is finite. By equations (2) and (3), the opposite trend of FR and MRL is obvious.

Let $G(t ; \alpha, \beta, k)$ be the cdf of the GG distribution [2] that is:

$$
G(t ; \alpha, \beta, k)=\frac{\gamma\left(k,\left(\frac{t}{\alpha}\right)^{\beta}\right)}{\Gamma(k)},
$$

in which $\alpha>0, \beta>0, k>0, \gamma(k, x)=\int_{0}^{x} \omega^{k-1} e^{-\omega} d \omega$ is the incomplete gamma function and $\Gamma($.$) is the gamma$ function. For properties of GG see Lawless [31]. The cdf of KumGG distribution is defined by substituting the $G(t ; \alpha, \beta, k)$ in equation (1). Therefore its reliability function is:

$$
\bar{F}(t)=\left(1-\left\{\gamma_{1}\left[k,\left(\frac{t}{\alpha}\right)^{\beta}\right]\right\}^{\lambda}\right)^{\phi}
$$

where $\gamma_{1}(k, x)=\gamma(k, x) / \Gamma(k)$. The corresponding pdf of KumGG distribution is:

$$
\begin{aligned}
f(t)= & \frac{\lambda \beta \phi}{\alpha \Gamma(k)}\left(\frac{t}{\alpha}\right)^{\beta k-1} \exp \left[-\left(\frac{t}{\alpha}\right)^{\beta}\right] \\
& \times\left\{\gamma_{1}\left[k,\left(\frac{t}{\alpha}\right)^{\beta}\right]\right\}^{\lambda-1}\left[1-\left\{\gamma_{1}\left[k,\left(\frac{t}{\alpha}\right)^{\beta}\right]\right\}^{\lambda}\right]^{\phi-1} .
\end{aligned}
$$

In model (4), $\alpha$ is scale parameter and $\beta, \lambda, \phi$ and $k$ are shape parameters. For more details about the properties of KumGG see Pascoa et al. [11]. Assuming $\phi=1$, the KumGG distribution reduces into EGG and the respective pdf is:

$$
\begin{aligned}
f(t)= & \frac{\lambda \beta}{\alpha \Gamma(k)}\left(\frac{t}{\alpha}\right)^{\beta k-1} \\
& \times \exp \left[-\left(\frac{t}{\alpha}\right)^{\beta}\right]\left\{\gamma_{1}\left[k,\left(\frac{t}{\alpha}\right)\right]\right\}^{\lambda-1} .
\end{aligned}
$$




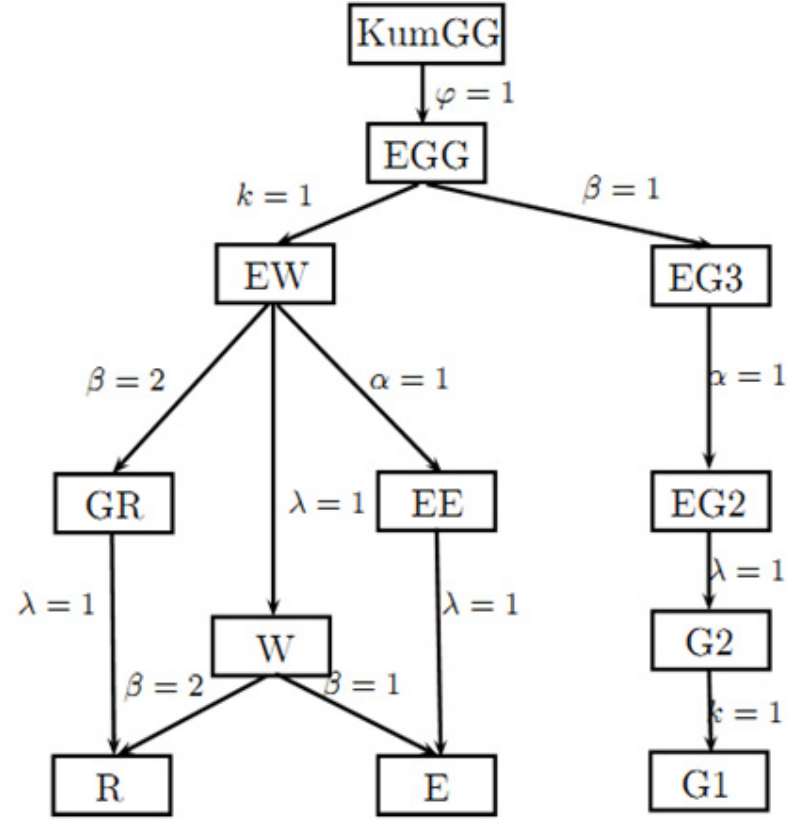

Fig. 1. The relationship of KumGG distribution and some of its important sub-models.

A real lifetime example is given by Cordeiro et al. [1] who found EGG as the appropriate distribution fitting to survival time of 112 patients after kidney transplant.

Moreover, by letting $\phi=\beta=1$ and $\phi=\beta=\alpha=1$ we have the EG3 and EG2 distribution functions respectively. Real dataset from Lawless [31] referring to the number of million revolutions before failure for each of 23 ball bearings in the life test, were fitted to a few lifetime distributions including EG3 by Gupta and Kundu [5].

The two-parameter gamma (G2) and one-parameter gamma (G1) are also obtained if $\phi=\beta=\lambda=1$ and $\phi=\beta=\lambda=k=1$.

Furthermore, EW and EE distributons are respectively obtained by assuming $\phi=k=1$ and $\phi=k=\alpha=1$. Also, GR distribution is acquired if $\beta=2$ in addition to $\phi=k=1$.

Cordeiro et al. [1] showed the relationships of EGG and all of its sub-models which contains a considerable number of beneficial distributions. Figure 1 demonstrates the relationships between KumGG, EGG and some of its commonly used sub-models. To consider all the sub-models accurately see $[1,11]$.

\section{$3 \mathrm{FR}$ and MRL shapes and change points}

KumGG distribution contains all increasing, decreasing, bathtub-shaped and unimodal failure rate models which depends on the values of its parameters. This characteristic is advantageous to estimate the individual failure rate functions and both relative failures and relative times [32].
Utilizing the definitions, FR and MRL functions for KumGG distribution respectively are

$$
\begin{aligned}
h(t)= & \frac{\lambda \beta \phi}{\alpha \Gamma(k)}\left(\frac{t}{\alpha}\right)^{\beta k-1} \exp \left[-\left(\frac{t}{\alpha}\right)^{\beta}\right]\left\{\gamma_{1}\left[k,\left(\frac{t}{\alpha}\right)^{\beta}\right]\right\}^{\lambda-1} \\
& \times\left(1-\left\{\gamma_{1}\left[k,\left(\frac{t}{\alpha}\right)^{\beta}\right]\right\}^{\lambda}\right)^{-1},
\end{aligned}
$$

and

$$
m(t)=\frac{\int_{t}^{\infty}\left(1-\left\{\gamma_{1}\left[k,\left(\frac{x}{\alpha}\right)^{\beta}\right]\right\}^{\lambda}\right)^{\phi} d x}{\left(1-\left\{\gamma_{1}\left[k,\left(\frac{t}{\alpha}\right)^{\beta}\right]\right\}^{\lambda}\right)^{\phi}}
$$

Different shapes of EGG failure rate function which involves analyzing its first derivative has been inspected in [1]. Let $h_{1}$ and $h_{2}$ respectively indicate KumGG and EGG failure rate functions, we have $h_{1}=\phi h_{2}$. Thus the same conditions for the shapes of EGG distribution are existing for the shapes of KumGG failure rate function. In this paper, we only focus on the change points of bathtub shaped FR functions therefore we need to have $\beta>1$ and $\beta \lambda<1$ (see $[1,3])$.

It is proved that where $\mathrm{FR}$ is differentiable bathtubshaped with unique change point $0<\tau_{0}<\infty$, if $h(0) m(0)>1$ then MRL is upside-down bathtub-shaped with a unique change point $t_{0}$ and satisfying $0<t_{0} \leqslant \tau_{0}$ (see [33]). Earlier, Mi [13] had also shown that the change point of bathtub-shaped failure rate is greater than or equal to the change point of its corresponding upside-down bathtub-shaped MRL. Guess et al. [34] proved the same theorem.

For some parameter values, the necessary condition to have $0<t_{0} \leqslant \tau_{0}$ is not always provided in bathtub-shaped FR functions of KumGG and its sub-models.

As it was stated, studying the bathtub-shaped FR function results considering the corresponding MRL function of an opposite trend. Therefore while determining the FR change point by minimizing the function leads to maximizing the MRL function to accurate its change point.

In Figure 2, some FR and MRL functions and their change points of KumGG distribution with different parameter values are illustrated. The solid points mark the locations of the change points. In these graphs change points of MRL occure before FR change points, though in KumGG distribution it is not a necessary condition. Besides, by comparing the parameters with greater values of $k$ not only the change points distances increase, but also MRL maximum takes larger values.

\section{Length of the useful period}

For bathtub-shaped failure rate models the length of stable period is an important feature since in reality greater constant region grants a longer time to apply the unit. 

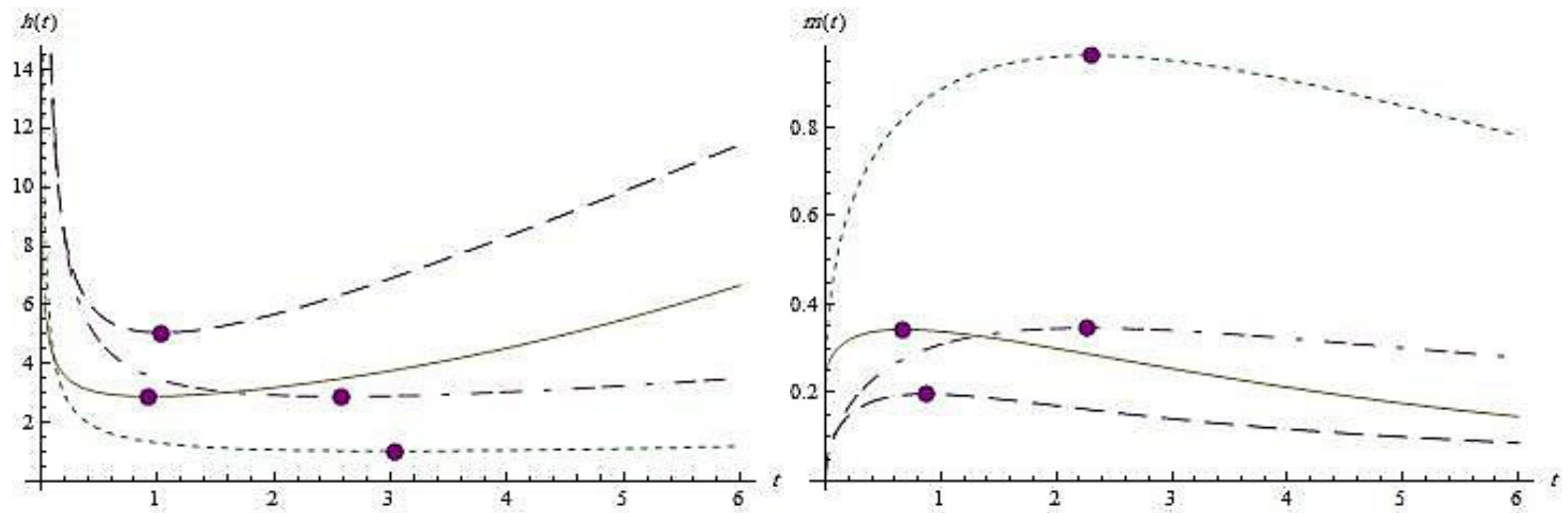

Fig. 2. Left: FR function of KumGG distributions; right: MRL function of KumGG distributions. " $--": k=0.1, \alpha=2, \beta=$

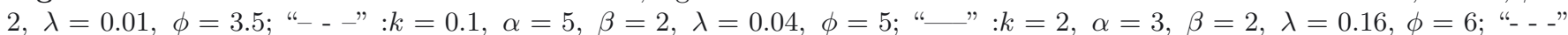
$: k=2.6, \alpha=5, \beta=2, \lambda=0.04, \phi=3$.

To derive the optimum burn-in time it is a reasonable method to find the point which maximizes the MRL function, see Weiss and Dishon [35]. There are other authors with different point of views on burn-in time, such as Yun et al. [36], determining the optimal time by minimizing the total mean cost. Considering the maximum of MRL as the optimum burn-in time results studying the length of useful period after the burn-in time [26].

Hence for the bathtub-shaped FR models of the generalized gamma-type distributions which were previously introduced, we study the length between the minimum of FR and maximum of MRL functions as the useful period: Length $=\tau_{0}-t_{0}$. Besides consider the relative difference $D=$ Length $/ \tau_{0}$ which could be considered as a scale-free measure to compare the useful periods. The larger distance provides a longer stable period so FR function is flat and curve does not rapidly change its direction.

In Tables $1-5$, the FR and MRL change points, their differences and the relative difference for various parameter values of EG2, EG3, EGG, and KumGG distribution functions are presented. In the last column of the tables, the stared values demonstrate the $D>0.9$, which is considerably a large relative difference between the change points.

Apparently the FR and MRL functions change points and their differences are highly related to the values of model parameters. In all four employed distributions, increasing the value of $k$ results larger length and averagely greater $D$. This effect is better clear while $k>1$. The other shape parameter $\lambda$ has no clear effect on $D$ for EG2 and EG3 while $0<k<1$, but as soon as $k>1$, a slight increasing influence of greater $\lambda$ could be recognized. Investigating the effect of $\lambda$ in EGG and KumGG, the direct increasing effect of this parameter for any value of $k$ is obviuos.

In EG3, EGG and KumGG distributions, the scale parameter $\alpha$ has no specific effect on the length or $D$. Considering different values of $\alpha$ for fixed values of $k$ and $\lambda$ in EG3, in addition fixed $\beta$ in EGG and fixed $\phi$ for KumGG distributions, make no difference in the change points and also their distances. The new shape parameter, $\beta$, in EGG
Table 1. Change points and their differences of EG2.

\begin{tabular}{cccccc}
\hline$k$ & $\lambda$ & $\tau_{0}$ & $t_{0}$ & Length & $D$ \\
\hline 0.1 & 0.01 & 20.0903 & 20.0903 & 0 & 0 \\
0.1 & 0.04 & 8898.8467 & 5.1931 & 8893.6543 & $0.9994^{*}$ \\
0.1 & 0.07 & 8898.8467 & 5.1931 & 8893.6543 & $0.9994^{*}$ \\
0.1 & 0.1 & 20.0903 & 20.0903 & 0 & 0 \\
0.1 & 0.13 & 8898.8467 & 5.1931 & 8893.6543 & $0.9994^{*}$ \\
0.1 & 0.19 & 20.0903 & 20.0903 & 0 & 0 \\
0.4 & 0.01 & 20.0903 & 20.0903 & 0 & 0 \\
0.4 & 0.04 & 27.5546 & 20.0903 & 7.4643 & 0.2709 \\
0.4 & 0.07 & 24.1917 & 23.8535 & 0.3383 & 0.014 \\
0.4 & 0.1 & 20.0903 & 27.5338 & -7.4435 & -0.3705 \\
0.4 & 0.13 & 118.3107 & 10.1588 & 108.1519 & $0.9141^{*}$ \\
0.4 & 0.16 & 34.9876 & 5.1931 & 29.7945 & 0.8516 \\
0.7 & 0.01 & 23.9822 & 20.0903 & 3.8919 & 0.1623 \\
0.7 & 0.04 & 20.0903 & 26.7617 & -6.6713 & -0.3321 \\
0.7 & 0.07 & 29.4045 & 20.0903 & 9.3142 & 0.3168 \\
0.7 & 0.1 & 20.0903 & 27.6931 & -7.6028 & -0.3784 \\
0.7 & 0.13 & 25.735 & 25.056 & 0.6791 & 0.0264 \\
0.7 & 0.16 & 8898.8467 & 10.1588 & 8888.6885 & $0.9989^{*}$ \\
0.7 & 0.19 & 8898.8467 & 10.1588 & 8888.6885 & $0.9989^{*}$ \\
2.5 & 0.01 & 3.3412 & 2.5446 & 0.7966 & 0.2384 \\
2.5 & 0.04 & 3.2267 & 2.4307 & 0.7959 & 0.2467 \\
2.5 & 0.07 & 3.1043 & 2.3095 & 0.7948 & 0.256 \\
2.5 & 0.1 & 2.9729 & 2.1798 & 0.7931 & 0.2668 \\
2.5 & 0.13 & 2.831 & 2.0403 & 0.7907 & 0.2793 \\
2.5 & 0.16 & 2.6768 & 1.8895 & 0.7873 & 0.2941 \\
2.5 & 0.19 & 2.5078 & 1.7254 & 0.7824 & 0.312 \\
2.8 & 0.01 & 3.3436 & 2.506 & 0.8376 & 0.2505 \\
2.8 & 0.04 & 3.2122 & 2.3753 & 0.8368 & 0.2605 \\
2.8 & 0.07 & 3.0706 & 2.2352 & 0.8354 & 0.2721 \\
2.8 & 0.1 & 2.9173 & 2.0841 & 0.8332 & 0.2856 \\
2.8 & 0.13 & 2.75 & 1.9203 & 0.8297 & 0.3017 \\
2.8 & 0.16 & 2.5656 & 1.7412 & 0.8244 & 0.3213 \\
2.8 & 0.19 & 2.3603 & 1.5439 & 0.8164 & 0.3459 \\
\hline & & & & &
\end{tabular}

and KumGG distributions has a direct increasing influence on the difference as it takes greater values. However, studying the extera shape parameter $\phi$ in KumGG distribution decreases the length and also the relative difference, since increasing the value of $\phi$ does not alter FR 
Table 2. Change points and their differences of EG3.

\begin{tabular}{ccccccc}
\hline$k$ & $\alpha$ & $\lambda$ & $\tau_{0}$ & $t_{0}$ & Length & $D$ \\
\hline 0.1 & 2 & 0.01 & 39.9533 & 39.9533 & 0 & 0 \\
0.1 & 2 & 0.04 & 6356.3843 & 10.1588 & 6346.2256 & $0.9984^{*}$ \\
0.1 & 2 & 0.07 & 39.9533 & 39.9533 & 0 & 0 \\
0.1 & 2 & 0.1 & 39.9533 & 39.9533 & 0 & 0 \\
0.1 & 2 & 0.13 & 6356.3843 & 10.1588 & 6346.2256 & $0.9984^{*}$ \\
0.1 & 2 & 0.16 & 39.9533 & 39.9533 & 0 & 0 \\
0.1 & 2 & 0.19 & 39.9533 & 43.988 & -4.0347 & -0.101 \\
0.1 & 3 & 0.01 & 79.6793 & 79.6793 & 0 & 0 \\
0.1 & 3 & 0.04 & 6356.3843 & 20.0903 & 6336.2939 & $0.9968^{*}$ \\
0.1 & 3 & 0.07 & 79.6793 & 66.0234 & 13.6559 & 0.1714 \\
0.1 & 3 & 0.1 & 68.5066 & 63.9686 & 4.538 & 0.0662 \\
0.1 & 3 & 0.13 & 6356.3843 & 20.0903 & 6336.2939 & $0.9968^{*}$ \\
0.1 & 3 & 0.16 & 70.8347 & 66.1783 & 4.6563 & 0.0657 \\
0.1 & 3 & 0.19 & 72.3091 & 67.4928 & 4.8163 & 0.0666 \\
0.7 & 2 & 0.01 & 39.9533 & 45.3167 & -5.3633 & -0.1342 \\
0.7 & 2 & 0.04 & 50.4958 & 39.9533 & 10.5425 & 0.2088 \\
0.7 & 2 & 0.07 & 57.0259 & 39.9533 & 17.0726 & 0.2994 \\
0.7 & 2 & 0.1 & 51.883 & 54.8879 & -3.0049 & -0.0579 \\
0.7 & 2 & 0.13 & 39.9533 & 48.8337 & -8.8804 & -0.2223 \\
0.7 & 2 & 0.16 & 6356.3843 & 20.0903 & 6336.2939 & $0.9968^{*}$ \\
0.7 & 2 & 0.19 & 39.9533 & 39.9533 & 0 & 0 \\
0.7 & 5 & 0.01 & 79.6793 & 79.6793 & 0 & 0 \\
0.7 & 5 & 0.04 & 159.1313 & 124.9905 & 34.1407 & 0.2145 \\
2.8 & 3 & 0.01 & 10.0309 & 7.518 & 2.5129 & 0.2505 \\
2.8 & 3 & 0.04 & 9.6365 & 7.126 & 2.5105 & 0.2605 \\
2.8 & 3 & 0.07 & 9.2119 & 6.7056 & 2.5063 & 0.2721 \\
2.8 & 3 & 0.13 & 8.2499 & 5.7608 & 2.4891 & 0.3017 \\
2.8 & 3 & 0.19 & 7.0809 & 4.6317 & 2.4491 & 0.3459 \\
2.8 & 5 & 0.01 & 16.7182 & 12.5302 & 4.188 & 0.2505 \\
2.8 & 5 & 0.04 & 16.0608 & 11.8767 & 4.1841 & 0.2605 \\
2.8 & 5 & 0.07 & 15.3531 & 11.176 & 4.1771 & 0.2721 \\
2.8 & 5 & 0.13 & 13.7498 & 9.6013 & 4.1485 & 0.3017 \\
2.8 & 5 & 0.19 & 11.8014 & 7.7196 & 4.0818 & 0.3459 \\
\hline & & & & & & \\
\hline
\end{tabular}

change point while the MRL change point takes a larger value and also occurs nearer to the FR change point.

If just focus on the maximum of MRLs, they could reach to significantly large values in EG2 while $0<k<1$, although sometimes there is no difference between MRL and FR turning points. The very high values occur seldom if more shape parameters are included in the model, as you see in EG3 and specially in EGG distributions. Besides, there exist values of Length and $D$ which are less than 0 and that implies $\tau_{0}<t_{0}$. This unsatisfactory event only occurs for EG2 and EG3 distributions, specially as $0<k<1$. Therefore, increasing the number of shape parameters of the model can obviously avoid the inapproperiate situation. To have a better insight of parameters effects, see Figures 3-8.

On the left graph of Figure 3, it is demonstrated that in EG2 there is no clear effect of increasing $\lambda$ while $k<1$, but as soon as $k>1$, the graph on the right, the larger $\lambda$ results larger relative distance.

Figure 4 reveals the same conclusion as Figure 3 for EG3 distribution.
Table 3. Change points and their differences of EGG.

\begin{tabular}{cccccccc}
\hline$k$ & $\alpha$ & $\beta$ & $\lambda$ & $\tau_{0}$ & $t_{0}$ & Length & $D$ \\
\hline 0.1 & 2 & 4 & 0.01 & 0.6920 & 0.3619 & 0.3301 & 0.4771 \\
0.1 & 2 & 4 & 0.07 & 0.6821 & 0.3518 & 0.3303 & 0.4842 \\
0.1 & 2 & 4 & 0.16 & 0.6671 & 0.3367 & 0.3304 & 0.4953 \\
0.1 & 2 & 5 & 0.01 & 0.6814 & 0.3425 & 0.3389 & 0.4974 \\
0.1 & 2 & 5 & 0.07 & 0.6701 & 0.3307 & 0.3394 & 0.5065 \\
0.1 & 2 & 5 & 0.16 & 0.6527 & 0.3128 & 0.3399 & 0.5208 \\
0.7 & 2 & 5 & 0.01 & 0.7190 & 0.3516 & 0.3675 & 0.5111 \\
0.7 & 2 & 5 & 0.04 & 0.6748 & 0.3066 & 0.3683 & 0.5457 \\
0.7 & 2 & 5 & 0.07 & 0.6269 & 0.2597 & 0.3672 & 0.5858 \\
0.7 & 2 & 5 & 0.1 & 0.5745 & 0.2111 & 0.3634 & 0.6325 \\
0.7 & 2 & 5 & 0.13 & 0.5165 & 0.1614 & 0.3551 & 0.6875 \\
0.7 & 2 & 5 & 0.16 & 0.4513 & 0.1116 & 0.3398 & 0.7528 \\
0.7 & 2 & 5 & 0.19 & 0.3767 & 0.0334 & 0.3433 & 0.9114 \\
2.3 & 4 & 3 & 0.01 & 1.8696 & 0.9589 & 0.9107 & 0.4871 \\
2.3 & 4 & 3 & 0.04 & 1.5911 & 0.6986 & 0.8926 & 0.5610 \\
2.3 & 4 & 3 & 0.07 & 1.2693 & 0.4252 & 0.8441 & 0.6650 \\
2.3 & 4 & 3 & 0.1 & 0.8816 & 0.1607 & 0.7209 & 0.8178 \\
2.3 & 4 & 3 & 0.13 & 0.3696 & 0.0334 & 0.3362 & $0.9097^{*}$ \\
2.3 & 4 & 3 & 0.16 & 0.0334 & 0.0334 & 0.0000 & 0.0000 \\
2.3 & 4 & 3 & 0.19 & 0.0334 & 0.0334 & 0.0000 & 0.0000 \\
2.3 & 4 & 4 & 0.01 & 1.6353 & 0.7859 & 0.8494 & 0.5194 \\
2.3 & 4 & 4 & 0.04 & 1.3168 & 0.4870 & 0.8298 & 0.6302 \\
2.3 & 4 & 4 & 0.07 & 0.9059 & 0.1764 & 0.7295 & 0.8052 \\
2.6 & 5 & 2 & 0.01 & 3.4306 & 1.9433 & 1.4873 & 0.4335 \\
2.6 & 5 & 2 & 0.04 & 3.0288 & 1.5718 & 1.4570 & 0.4811 \\
2.6 & 5 & 2 & 0.07 & 2.5831 & 1.1804 & 1.4027 & 0.5430 \\
2.6 & 5 & 2 & 0.13 & 1.5216 & 0.3855 & 1.1361 & 0.7467 \\
2.6 & 5 & 2 & 0.16 & 0.8712 & 0.0749 & 0.7963 & $0.9140 *$ \\
2.6 & 5 & 3 & 0.01 & 2.3774 & 1.2093 & 1.1682 & 0.4914 \\
2.6 & 5 & 3 & 0.04 & 1.9698 & 0.8315 & 1.1384 & 0.5779 \\
2.6 & 5 & 3 & 0.07 & 1.4857 & 0.4359 & 1.0498 & 0.7066 \\
2.6 & 5 & 3 & 0.1 & 0.8724 & 0.0509 & 0.8215 & $0.9417^{*}$ \\
2.6 & 5 & 3 & 0.13 & 0.1886 & 0.0334 & 0.1552 & 0.8230 \\
2.6 & 5 & 3 & 0.16 & 0.1886 & 0.0334 & 0.1552 & 0.8230 \\
2.6 & 5 & 3 & 0.19 & 0.1886 & 0.0334 & 0.1552 & 0.8230 \\
\hline & & & & & & &
\end{tabular}

The lowest curve in Figure 5 belongs to $\beta=2$ and curves go to the upper locations when $\beta$ takes larger values in EGG distribution.

In Figure 6, the most important point is that for all values of $k$, the value of $\lambda$ has a direct effect on the value of $D$ in KumGG distribution.

The direct increasing effect of larger values of $\beta$ on the values of $D$ for KumGG is shown in Figure 7 .

Figure 8 illustrates that the value of $\alpha$ has no effect on the relative diffrence. It also shows the effect of larger $k$ which results larger $D$; because, in every parallel paire of lines the upper line belongs to the $k=1.3$ and the lower one refers to $k=0.1$. This difference will gradually decrease as soon as parameter $\phi$ takes larger values. Besides, the decreasing effect of $\phi$ is clear in shifting the horizontal trend lines to lower values on $D$ axis.

Ultimately, it is necessary to mention that some change points do not occur in the essential condition of $t>0$. 
Table 4. Change points and their differences of KumGG, $0<$ $k<1$.

\begin{tabular}{ccccccccc}
\hline$k$ & $\alpha$ & $\beta$ & $\lambda$ & $\phi$ & $\tau_{0}$ & $t_{0}$ & Length & $D$ \\
\hline 0.1 & 2 & 5 & 0.01 & 0.5 & 0.6814 & 0.2322 & 0.4492 & 0.6592 \\
0.1 & 2 & 5 & 0.01 & 2 & 0.6814 & 0.4536 & 0.2278 & 0.3343 \\
0.1 & 2 & 5 & 0.01 & 3 & 0.6814 & 0.51 & 0.1714 & 0.2515 \\
0.1 & 2 & 5 & 0.01 & 4 & 0.6814 & 0.5442 & 0.1372 & 0.2014 \\
0.1 & 2 & 5 & 0.01 & 5 & 0.6814 & 0.5671 & 0.1143 & 0.1677 \\
0.1 & 2 & 5 & 0.07 & 0.5 & 0.6701 & 0.2223 & 0.4478 & 0.6683 \\
0.1 & 2 & 5 & 0.07 & 2 & 0.6701 & 0.441 & 0.2291 & 0.3419 \\
0.1 & 2 & 5 & 0.07 & 3 & 0.6701 & 0.4973 & 0.1728 & 0.2578 \\
0.1 & 2 & 5 & 0.07 & 4 & 0.6701 & 0.5316 & 0.1385 & 0.2067 \\
0.1 & 2 & 5 & 0.07 & 5 & 0.6701 & 0.5546 & 0.1155 & 0.1723 \\
0.1 & 2 & 5 & 0.1 & 0.5 & 0.6644 & 0.2173 & 0.447 & 0.6729 \\
0.1 & 2 & 5 & 0.1 & 2 & 0.6644 & 0.4346 & 0.2298 & 0.3458 \\
0.1 & 2 & 5 & 0.1 & 3 & 0.6644 & 0.4909 & 0.1734 & 0.2611 \\
0.1 & 2 & 5 & 0.1 & 4 & 0.6644 & 0.5252 & 0.1391 & 0.2094 \\
0.1 & 2 & 5 & 0.1 & 5 & 0.6644 & 0.5483 & 0.1161 & 0.1747 \\
0.1 & 2 & 5 & 0.13 & 2 & 0.6586 & 0.4282 & 0.2304 & 0.3499 \\
0.1 & 2 & 5 & 0.13 & 3 & 0.6586 & 0.4844 & 0.1741 & 0.2644 \\
0.1 & 2 & 5 & 0.13 & 4 & 0.6586 & 0.5188 & 0.1398 & 0.2122 \\
0.1 & 2 & 5 & 0.13 & 5 & 0.6586 & 0.5419 & 0.1167 & 0.1771 \\
0.1 & 2 & 5 & 0.19 & 0.5 & 0.6468 & 0.2023 & 0.4445 & 0.6872 \\
0.1 & 2 & 5 & 0.19 & 2 & 0.6468 & 0.4151 & 0.2317 & 0.3582 \\
0.1 & 2 & 5 & 0.19 & 3 & 0.6468 & 0.4713 & 0.1755 & 0.2713 \\
0.1 & 2 & 5 & 0.19 & 4 & 0.6468 & 0.5057 & 0.1411 & 0.2181 \\
0.1 & 2 & 5 & 0.19 & 5 & 0.6468 & 0.5289 & 0.1179 & 0.1822 \\
\hline & & & & & & & &
\end{tabular}

Of course, these cases would never be naturally studied in real life; however, it could be advantagous to know that the occurence of these change points is so rare in EGG and never happens in KumGG. Therefore, by exponentiating GG and then EGG distributions, we approach to distributions of a higher capability of fitting to the real lifetime data.

\section{More investigation}

The effect of the models parameters on the relative distance $D$, specially the greater possible distances, has been discussed so far. It would be of great importance to consider the relative distance as it is $D=0$, which is obviously the direct result of Length $=0$. Here, we investigate this event in a general approach and look for the conditions in which the FR and MRL change points take equal values while not assuming any particular lifetime distributions, i.e., consider $h^{\prime}(t)=m^{\prime}(t)$ or equivalently $h(t)+c=m(t)$ where $c$ is a constant parameter. Consequently ought to solve the following equation

$$
\frac{f(t)}{\bar{F}(t)}+c=\frac{\int_{t}^{\infty} \bar{F}(x) d x}{\bar{F}(t)}, c \bar{F}(t)+f(t)=\int_{t}^{\infty} \bar{F}(x) d x
$$

Table 5. Change points and their differences of KumGG, $k>1$.

\begin{tabular}{ccccccccc}
\hline$k$ & $\alpha$ & $\beta$ & $\lambda$ & $\phi$ & $\tau_{0}$ & $t_{0}$ & Length & $D$ \\
\hline 2.3 & 2 & 5 & 0.01 & 1.5 & 0.7664 & 0.4294 & 0.337 & 0.4397 \\
2.3 & 2 & 5 & 0.01 & 2 & 0.7664 & 0.4833 & 0.2831 & 0.3694 \\
2.3 & 2 & 5 & 0.01 & 3 & 0.7664 & 0.5521 & 0.2142 & 0.2795 \\
2.3 & 2 & 5 & 0.01 & 4 & 0.7664 & 0.5943 & 0.1721 & 0.2246 \\
2.3 & 2 & 5 & 0.01 & 5 & 0.7664 & 0.6227 & 0.1437 & 0.1875 \\
2.3 & 2 & 5 & 0.04 & 0.5 & 0.5747 & 0.0881 & 0.4866 & 0.8466 \\
2.3 & 2 & 5 & 0.04 & 1.5 & 0.5747 & 0.2361 & 0.3386 & 0.5892 \\
3 & 2 & 2 & 0.01 & 0.5 & 1.4156 & 0.5655 & 0.8501 & 0.6005 \\
3 & 2 & 2 & 0.01 & 1.5 & 1.4156 & 0.9248 & 0.4907 & 0.3467 \\
3 & 2 & 2 & 0.01 & 2 & 1.4156 & 1.0101 & 0.4055 & 0.2864 \\
3 & 2 & 2 & 0.01 & 3 & 1.4156 & 1.1146 & 0.301 & 0.2126 \\
3 & 2 & 2 & 0.01 & 4 & 1.4156 & 1.1763 & 0.2393 & 0.169 \\
3 & 2 & 2 & 0.01 & 5 & 1.4156 & 1.217 & 0.1986 & 0.1403 \\
3 & 2 & 2 & 0.04 & 0.5 & 1.2221 & 0.4173 & 0.8049 & 0.6586 \\
3 & 2 & 2 & 0.04 & 1.5 & 1.2221 & 0.7353 & 0.4868 & 0.3983 \\
3 & 2 & 2 & 0.04 & 2 & 1.2221 & 0.8152 & 0.4069 & 0.333 \\
3 & 2 & 2 & 0.04 & 3 & 1.2221 & 0.9155 & 0.3066 & 0.2509 \\
3 & 2 & 2 & 0.04 & 4 & 1.2221 & 0.976 & 0.2461 & 0.2014 \\
3 & 2 & 2 & 0.04 & 5 & 1.2221 & 1.0166 & 0.2055 & 0.1682 \\
3 & 2 & 2 & 0.07 & 0.5 & 1.0032 & 0.2681 & 0.7351 & 0.7328 \\
3 & 2 & 2 & 0.07 & 1.5 & 1.0032 & 0.53 & 0.4732 & 0.4717 \\
3 & 2 & 2 & 0.07 & 2 & 1.0032 & 0.6012 & 0.402 & 0.4008 \\
3 & 2 & 2 & 0.07 & 3 & 1.0032 & 0.6937 & 0.3095 & 0.3085 \\
3 & 2 & 2 & 0.07 & 4 & 1.0032 & 0.7515 & 0.2517 & 0.2509 \\
3 & 2 & 2 & 0.07 & 5 & 1.0032 & 0.791 & 0.2122 & 0.2116 \\
3 & 2 & 2 & 0.1 & 0.5 & 0.7525 & 0.1283 & 0.6241 & 0.8294 \\
3 & 2 & 2 & 0.1 & 2 & 0.7525 & 0.37 & 0.3825 & 0.5083 \\
3 & 2 & 2 & 0.1 & 3 & 0.7525 & 0.4484 & 0.3041 & 0.4041 \\
3 & 2 & 2 & 0.1 & 4 & 0.7525 & 0.4999 & 0.2526 & 0.3357 \\
\hline & & & & & & & & \\
& & & & & & & & \\
3
\end{tabular}

differentiating (5) we have

$$
-c f(t)+f^{\prime}(t)=-\bar{F}(t),
$$

letting $\bar{F}(t)=y$ the below differential equation is resulted

$$
y^{\prime \prime}-c y^{\prime}+y=0
$$

to solve above differential equation we have

$$
\begin{aligned}
z^{2}-c z+1 & =0 \\
z & =\frac{1}{2}\left(c \pm \sqrt{c^{2}-4}\right) .
\end{aligned}
$$

The answer of (6) is conditioned on the delta's value:

1. If $c^{2}-4>0$ then $z=\frac{c_{1} \pm c_{2}}{2}$ which $c_{1}$ and $c_{2}$ are conctant parameters, therefore

$$
\bar{F}(t)=r_{1} e^{\frac{c_{1}+c_{2}}{2} t}+r_{2} e^{\frac{c_{1}-c_{2}}{2} t}
$$

while $r_{1}$ and $r_{2}$ are constant parameters

2. If $c^{2}-4=0$ then $z=\frac{1}{2} c$ and

$$
\bar{F}(t)=\left(r_{1}+r_{2} t\right) e^{\frac{c}{2} t}
$$

where $r_{1}$ and $r_{2}$ are constant parameters; 

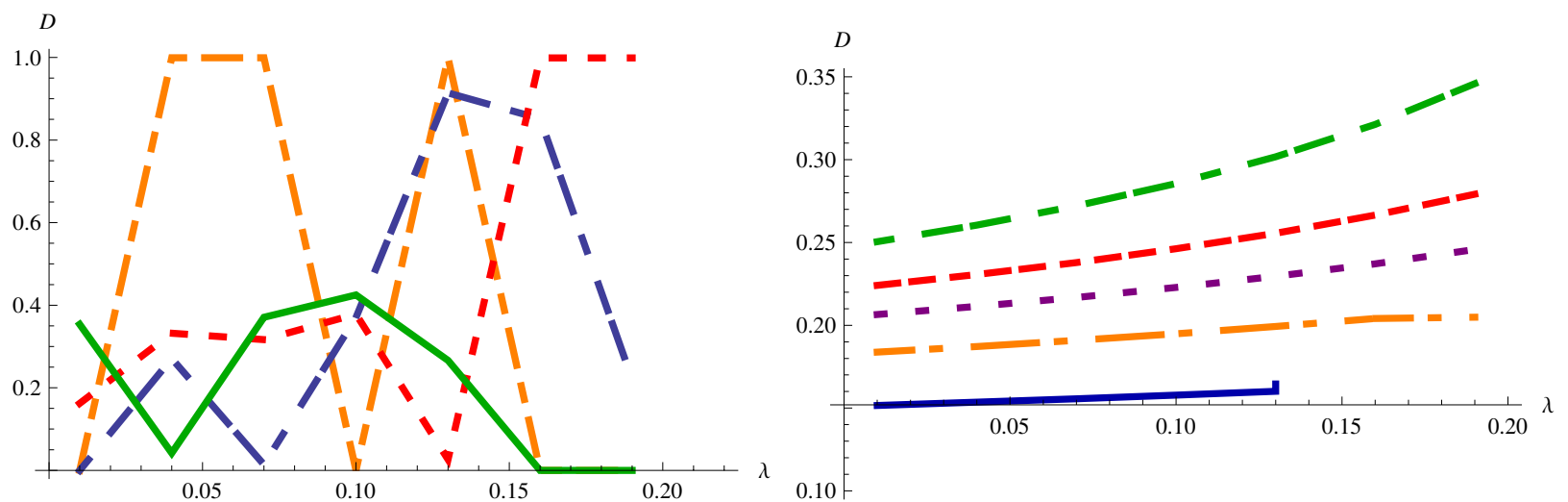

Fig. 3. The trend of $D$ for different values of $\lambda$ in EG2 distribution. Left: "- - -": $k=0.1 ;$ " $---": k=0.4 ; "--$ " : $k=0.7$;

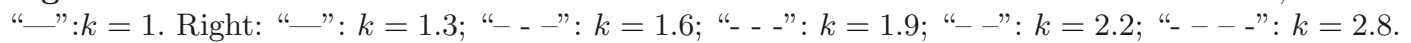
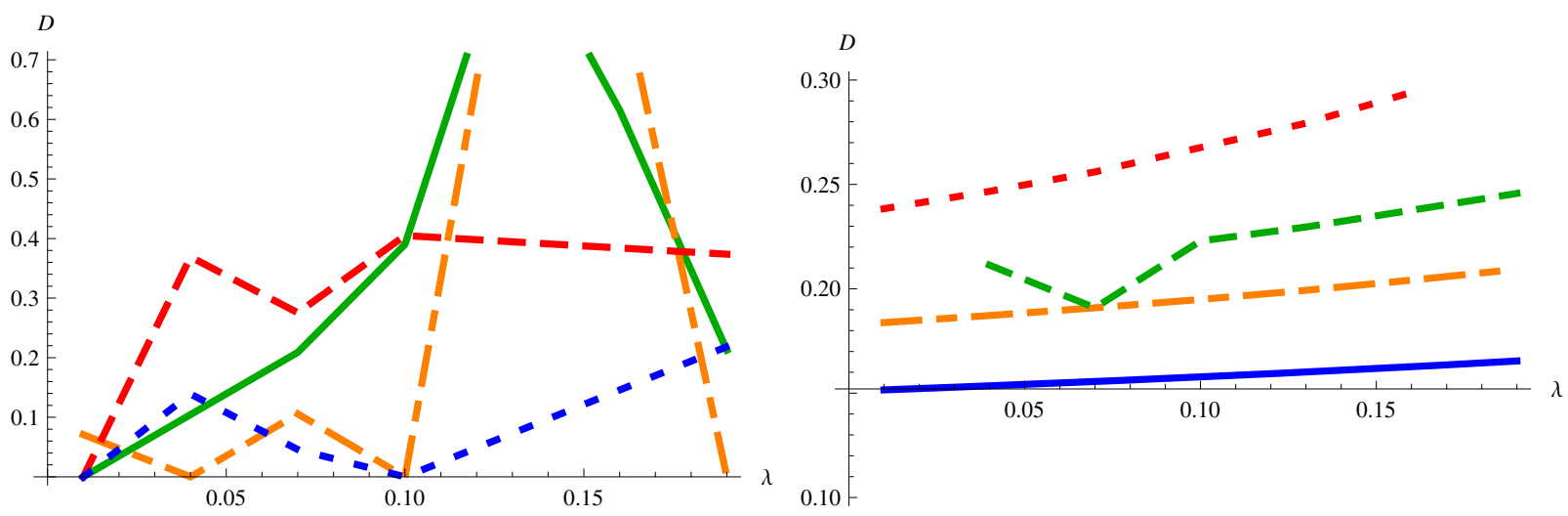

Fig. 4. The trend of $D$ for different values of $\lambda$ in EG3 distribution. Left: In all graphs $k=0.4$ and " —" $: \alpha=2 ; "-\ldots ": \alpha=3$;

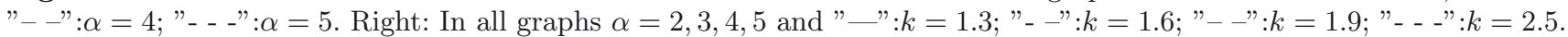

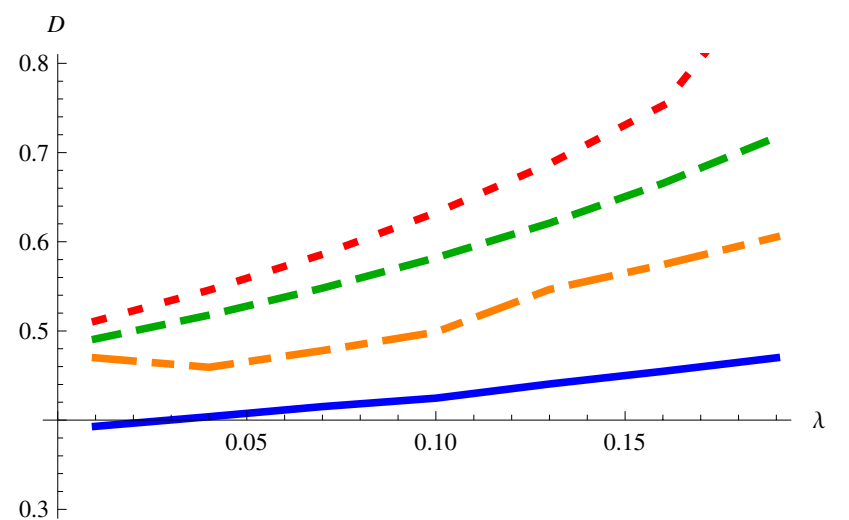

Fig. 5. The trend of $D$ for different values of $\lambda$ in EGG distribution. In all graphs $k=0.7, \alpha=2,3,4,5$ and "-" : $\beta=2$; " $-": \beta=3 ; "$ - " $: \beta=4 ; "$ - - -": $\beta=5$.

3. If $c^{2}-4<0$ then $z=a+i b$ where $a$ and $b$, respectively, are real and imaginary partials, so

$$
\bar{F}(t)=e^{a t}[A \cos (b t)+B \sin (b t)],
$$

in which $A$ and $B$ are constant parameters.

Considering the $D=0$ values and corresponding $\bar{F}(t)$ models, one can conclude that the second type of conditioined models is mostly occurred for EG2 and EG3 distributions.

\section{Conclusion}

This paper analysed the distance between the FR and MRL change points in KumGG, EGG, EG3, and EG2 as the useful period of these lifetime distributions which provides a guaranteed working period of units or systems lifetimes following the aforementioned distributions. Generally speaking, the larger the shape parameters are, the longer the distance will be. Consequently, units which could be modeled with larger shape parameters are expected to live longer whereas varying scale parameter in EG3, EGG and KumGG makes no change on the useful period. Besides, investigating the general model of distribution functions in which FR and MRL change points coincide, led to three conditioned distribution models and explained why EG2 and EG3 distributions for some particular parameter values offer no useful period.

If any parameter setting could be possible, decreasing the number of shape parameters and specifying particular range of $k(k>1)$ can result considerably long useful period and large MRL maximum. 

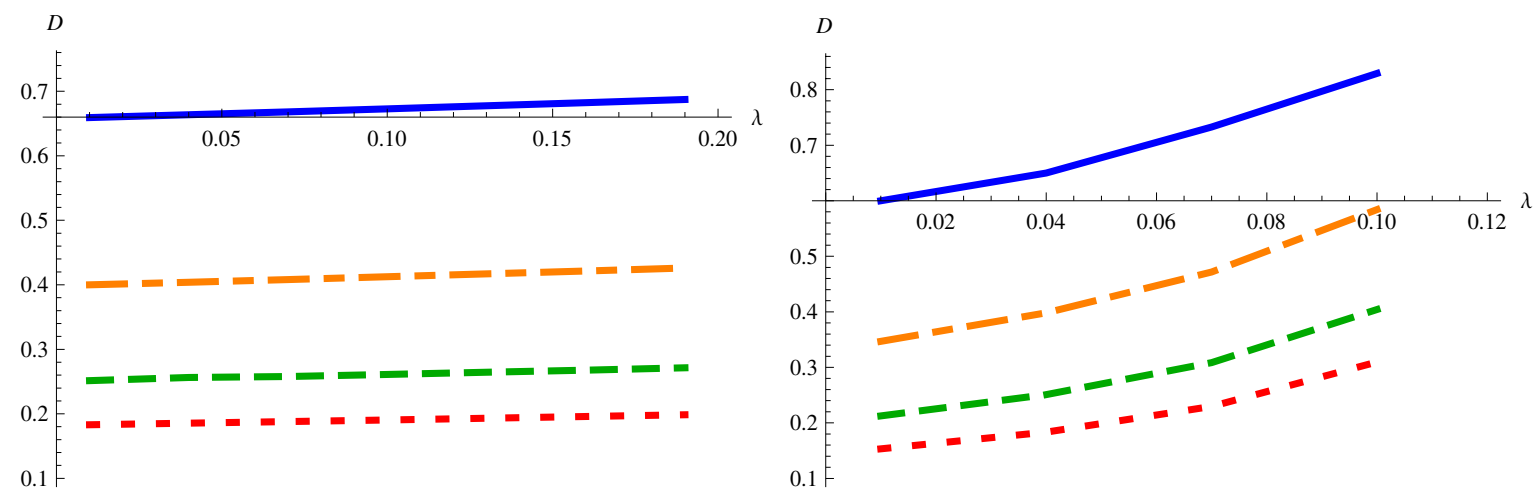

Fig. 6. The trend of $D$ for different values of $\lambda$ in KumGG distribution. Left: In all graphs $k=0.1, \alpha=2, \beta=5$ and " - ":

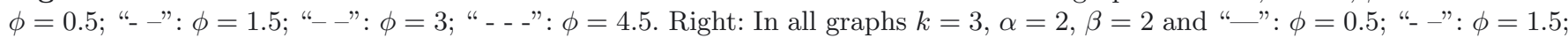
"- -": $\phi=3 ; "$; - -": $\phi=4.5$.

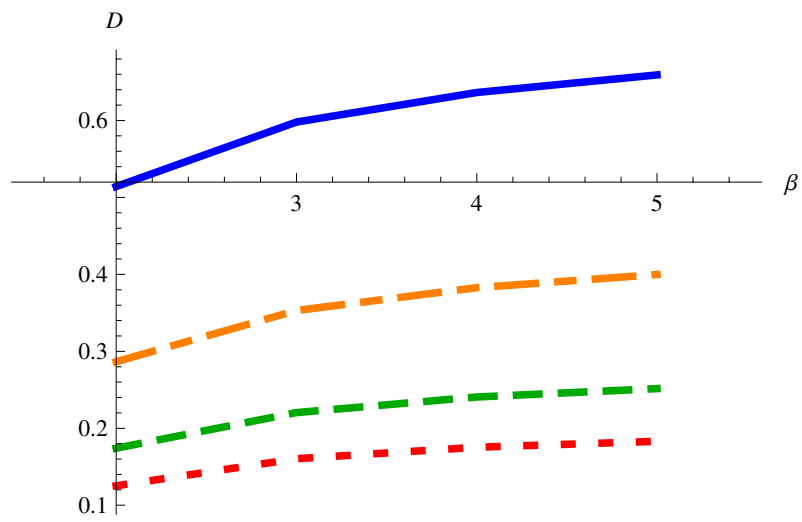

Fig. 7. The trend of $D$ for different values of $\beta$ in KumGG distribution. In all graphs $k=0.1, \alpha=2, \lambda=0.01$ and "- ":

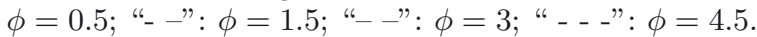

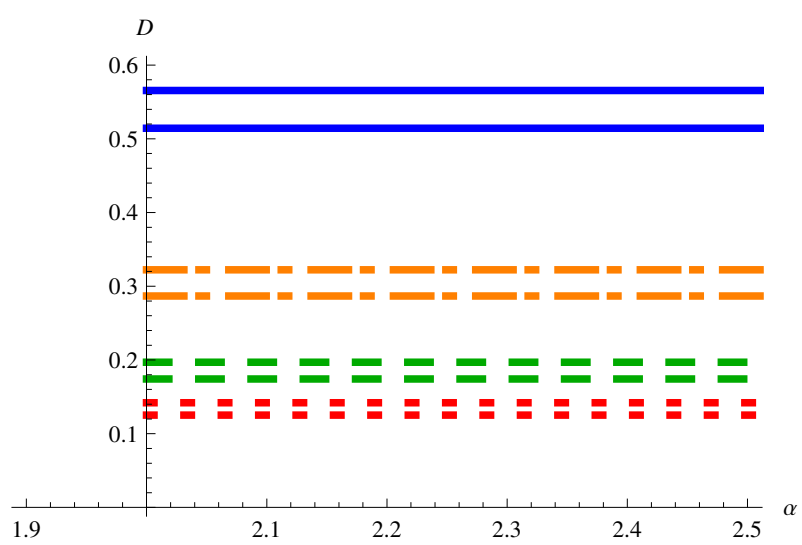

Fig. 8. The trend of $D$ for different values of $\alpha$ in KumGG distribution. In all graphs for the upper lines $k=1.3$ and for the lower lines $k=0.1$; in both $\beta=2, \lambda=0.01$ and "-": $\phi=0.5 ; "--": \phi=1.5 ; "-\_": \phi=3 ; "-. . ": \phi=4.5$.

Acknowledgements. The authors are grateful to the valuable recommendations of the referees which helped to improve this paper. Besides, the support of Ordered and Spatial Data Center of Excellence at Ferdowsi University of Mashhad is highly appreciating.

\section{References}

1. G.M. Cordeiro, E.M.M. Ortega, G.O Silva, The exponentiated generalized gamma distribution with application to lifetime data, J. Statist. Comput. Simul. iFirst, 1-16 (2010)

2. E.W. Stacy, A Generalization of the Gamma distribution, Ann. Math. Stat. 33, 1187-1192 (1962)

3. G.S. Mudholkar, D.K. Srivastava, M. Friemer, The exponentiated Weibull family: A reanalysis of the bus-MotorFailure data, Technometrics 37, 436-445 (1995)

4. G.S. Mudholkar, D.K. Srivastava, G.D. Kollia, A generalization of the Weibull distribution with the application to the analysis of survival data, J. Am. Statist. Assoc. 91, 1575-1583 (1996)

5. R.D. Gupta, D. Kundu, Generalized exponential distributions, Aust. N.Z. J. Stat. 41, 173-188 (1999)

6. R.D. Gupta, D. Kundu, Exponential exponentiated distribution: An alternative to Gamma and Weibull distributions, Biometrika J. 43, 117-130 (2001)

7. D. Kundu, M.Z. Raqab, Generalized Rayleigh distribution: Different methods of estimation, Comput. Stat. Data Anal. 49, 187-200 (2005)

8. P. Kumaraswamy, A generalized probability density function for double-bounded random-process, J. Hydrol. 462, 79-88 (1980)

9. M.C. Jones, Kumaraswamy's distribution: A beta-type distribution with some tractibility advantages, Staist. Met. 6, 70-81 (2009)

10. G.M. Cordeiro, M. de Castro, A new family of generalized distributions, J. Statist. Comput. Simul. 0, 1-17 (2009)

11. M.A.R. Pascoa, E.M.M. Ortega, G.M.C. Cordeiro, P.F. Paranaiba, The Kumaraswamy-generalized gamma distribution with application in survival analysis, Stat. Met. 8, 411-433 (2011)

12. R.C. Gupta, H.O. Akman, Mean residual life functions for certain types of non-monotonic ageing, Comm. Statist. Stochastic Models 11, 219-225 (1995)

13. J. Mi, Bathtub failure rate and upside-down bathtub mean residual life, IEEE Trans. Rel. 44, 388-391 (1995)

14. J.H. Lim, D.H. Park, Trend change in mean residual life, IEEE Trans. Rel. 44, 291-296 (1995)

15. M. Mitra, S.K. Basu, Change point estimation in nonmonotonic ageing models, Ann. Inst. Stat. Math. 47, 483491 (1995) 
16. A.A. Salvia, Some results on discrete mean residual life, IEEE Trans. Rel. 45, 359-361 (1996)

17. F. Guess, F. Proschan, Mean Residual Life: Theory and Applications (Handbook of Statistics 7: Quality Control and Reliability, North-Holland, 1988), pp. 215-224

18. G.L. Ghai, J. Mi, Mean residual life and its association with failure rate, IEEE Trans. Rel. 48, 262-266 (1999)

19. L.C. Tang, Y. Lu, E.P. Chew, Mean residual life of lifetime distributions, IEEE Trans. Rel. 48, 73-78 (1999)

20. Z.M. Chen, D. Ferger, J. Mi, Estimation of the change point of a distribution based on the number of failed test items, Metrika 53, 31-38 (2001)

21. L. Bekker, J. Mi, Shape and crossing properties of mean residual life functions, Statist. Probab. Lett. 64, 225-234 (2003)

22. J. Mi, A General approach to the shape of failure rate and MRL functions, Naval Res. Logist. 51, 543-556 (2004)

23. M. Bebbington, C.D. Lai, R. Zitikis, Estimating the turning point of a bathtub shaped failure distribution, J. Statist. Plann. Inference 138, 1157-1166 (2008)

24. Y. Shen, M. Xie, C.D. Tang, On the change point of the mean residual life of series and parallel systems, Aust. N. Z. J. Stat. 52, 109-121 (2010)

25. M. Shafaei Noughabi, G.R. Mohtashami Borzadran, A.H. Rezaei Roknabadi, On the reliability properties of some weighted models of bathtub shaped hazard rate distribution, Probab. Eng. Inform. Sci. 27, 125-140 (2013)

26. M. Xie, T.N. Goh, Y. Tang, On the changing point of mean residual life and failure rate function for generalized Weibull distributions, Reliab. Eng. Syst. Safe. 84, 293-299 (2004)
27. M. Bebbington, C.D. Lai, R. Zitikis, Useful period for lifetime distributions with bathtub shaped hazard rate functions, IEEE Trans. Rel. 55, 245-251 (2006)

28. M. Bebbington, C.D. Lai, R. Zitikis, Optimum burnin time for a bathtub shaped failure rate distribution, Methodol. Comput. Appl. Probab. 55, 245-251 (2007)

29. E.J. Muth, The Theory and Applications of Reliability, Reliability Models with Positive Memory Derived from the Mean Residual Life Function (Academic Press, London, 1977), Vol. 2, pp. 401-435

30. R. Calabria, G. Pulcini, On the maximum likelihood and least squares estimation in the inverse Weibull estimation, Statist. Appl. 2, 53-66 (1990)

31. J.F. Lawless, Statistical Models and Methods for Lifetime Data (Wiley, New York, 2003)

32. C. Cox, The generalized F distribution: an umberella for parametric survival analysis, Stat. Med. 27, 4301-4312 (2008)

33. C.D. Lai, M. Xie, Stochastic Ageing and Dependence for Reliability (Springer, New York, 2006)

34. F. Guess, K.H. Nam, D.H. Park, Failure rate and mean residual life with trend changes, Asia Pac. J. Oper. Res. 15, 239-244 (1998)

35. G.H. Weiss, M. Dishon, Some economic problems related to burn-in programs, IEEE Trans. Rel. 20, 190-195 (1971)

36. W.Y. Yun, Y.W. Lee, L. Ferreira, Optimal burn-in time under cumulative free replacement warranty, Reliab. Eng. Syst. Safety 78, 93-100 (2002) 